\title{
A Computational Approach to Multi-Level Analysis of Network Resilience
}

\author{
Christian Doerr and Javier Martin Hernandez \\ TU Delft, Department of Telecommunication, Mekelweg 4, 2628CD Delft, The Netherlands \\ Tel: +31 15 278-\{2279,2132\}, E-Mail: \{C.Doerr, J.MartinHernandez\}@ tudeflt.nl
}

\begin{abstract}
As communication and network technology has advanced into all areas of human life, large-scale failures of such infrastructures can have a drastic impact on its users, the economy and society in general. Significant attention has been paid to study and improve the dependability of such systems, intending to augment their resilience against challenges. This paper presents a computational approach for a multi-level multi-dimensional study of network robustness, providing hard performance guarantees for networks and helping to assess risk and uncertainty associated with threats. We demonstrate in a case study that such approach is well suited to understood the root causes and subsequent effects of network challenges and therefore obtain deeper insights into the resilience of a network under stress.
\end{abstract}

Keywords - Dependability assessment tools; dependability simulation; network planning

\section{INTRODUCTION}

With the proliferation and penetration of communication technology into all areas of human life, networks such as the Internet have become a normality and necessity, contributing and enabling to a large extent our current quality of life. Large scale outages like the great Northeastern blackout of 2003, the AS7007 blackhole [1], the Pakistan Telecom-YouTube [2] or most recent Google incidents however indicated that even small or locally contained causes may have a large scale regional and in some instances even global impact on infrastructures, creating significant damages to a company, country or society in general [3].

It is not surprising that in the recent past, the study of resilience of critical infrastructures has therefore received significant attention, with a number of projects and initiatives intending to investigate, measure and reduce the impact of accidental and intentional challenges on the performance of complex networks, and the Internet in particular. Of these investigations, a large body has focused on specific structural analyses of certain network topology paradigms (for the scale-free topology of the Internet see for example [4]), the general vulnerability of protocols (for BGP vulnerabilities see for example [5], [6]), metrics to quantify certain aspects of network topologies (for an overview see [7]) and most recently, resilience evaluations of concrete infrastructures through case studies for selected metrics (as for example [8]).

While these studies have provided insights into the causes for specific infrastructure failures and generated probabilistic risk and performance assessments of real-world networks such as the Sprint or Nobel network with respect to a particular metric such as availability, a deeper understanding into which topological features contribute to the overall resilience of infrastructures is still missing. If developed, such knowledge would enable network designers and operators to unerringly create, develop and enhance communication infrastructures that resiliently conform and fulfill service level requirements even under perturbations, such as equipment failures, natural disasters or intentional attacks.

Cholda et al. [9] conclude that this unsatisfactory situation may be attributed to the fact that current work on resilience is driven in an isolated and uncoordinated manner. We further argue that in order to obtain the best insights into what drives the resilience of networks (which may finally lead to an universal theory of robustness engineering), it will be also necessary for researchers to investigate the performance and response patterns of infrastructures under stress using a multilevel, multi-dimensional approach, monitoring and studying the behavior of networks both across a number of different, preferably orthogonal metrics as well as at different layers of the protocol stack and service architecture at the same time.

In this paper, we present a computational framework intended to help address these issues, which provides an open platform to combine and merge previous and ongoing work in the area of resilient networking, implementing already a significant body of previously published work in the areas of structural network analysis, topology generation and network metrics. Designed to either automatically explore or allow researchers to interactively investigate through a series of assisted "what-if" questions, we believe that the pooling of available proposals and tools from the community will provide the best route to get to an understanding of a network's resilience. The framework has been successfully applied in a series of case studies.

The contributions of this work are threefold: First, we present the first unified computational framework that is able to assess a network's resilience through a combination of structural topological analysis, network simulation and hardware emulation by relating a variety of metric results horizontally within each layer of the networking stack and vertically across different layers. Second, we introduce a set of simple, but yet very effective algorithmic and computational enhancements that make it possible to achieve a full exploration of the network state space. In contrast to previous work, this enables us to derive hard guarantees for network resilience, i.e., we can provide provable performance bounds that cannot be 
underscored by any type of attack or challenge. Yet, the resulting computational demands are comparable to current state-of-the-art mathematical programming approaches. Third, we introduce the concept of a robustness uncertainty area and discuss how network resilience may be best characterized through context- and challenge-specific robustness envelopes.

This paper is structured as follows: Section II gives a brief overview over the related work. Section III describes a series of considerations how to measure robustness, threats and uncertainty using envelopes. Section IV discusses the architecture and some implementation considerations of our computational framework. Section V presents a selection of results obtained in a resilience case study of the European research network GEANT2. Section VI summarizes and concludes our work.

\section{RELATED WORK}

A wealth of procedures to evaluate and improve network robustness have been proposed over the last 50 years. The first approach to network robustness was based on the life cycle of a network component, namely the mean time between failures (MTBF). Further, scientists studied the probability of a graph to remain connected after a number of network component failures by using graph percolation properties [10], [11] and reliability polynomials [12], [13]. Recent attention has been given to the study of power law networks reliability [14]-[16] since Faloutsos et al. showed that the Internet topology degree distribution follows a power law [17]. Reliability studies are used to investigate the risk for a network to become disconnected by making use of stochastic models, but presen two drawbacks. First, these studies ignore the multi-level service nature of networks and cannot be regarded as complete models, an second, reliability studies have been proven not to be optimal due to the irregular stress cycles of network elements [18].

Due to the need to improve the resilience level of critical infrastructures a number of international projects have been presented during the last decade. Examples of this are the international projects GRID, AMBER, HIDENETS, ResiliNets, the network of excellence ReSIST, etc. The mentioned projects provide important advances in their respective fields (power grids and computing systems), proposing techniques and algorithms to improve system evolvability, assessability, usability or diversity [19].

However the majority of these studies focus on specific systems, e.g. SQL database software, lacking the generality of a multidisciplinary framework. E.g. GRID studies power systems vulnerabilities, in view of the challenges driven by the transformation of the European power infrastructure, ReSIST leads research activities to ensure that present and future computing systems have the necessary resilience and survivability.

In recent years network vulnerability has been a target of focused study. The response of complex networks subject to any type of attacks have been investigated [15], [20] in order to quantify network robustness. Most of the studies focus on simulating challenge scenarios by removing links from a graph in a random fashion, while tracking the behavior through a set of graph metrics (e.g. connectivity, average hopcount, betweenness, etc.). These studies provide valuable insights on the presented metrics, however they do not succeed to provide a general definition of network robustness. Furthermore exact robustness levels cannot be guaranteed from these studies: give the stochastic nature of the analysis only asymptotic bounds can be provided.

\section{BENCHMARKING ROBUSTNESS, THREATS AND UNCERTAINTY}

Building upon and bringing together various previous proposals to assess the performance of a networking system, this particular work aims at assessing and benchmarking the robustness of a network, in other words the change in metrics and their sensitivity when the network is encountering stress, as caused by equipment failures, natural disasters or intentional attacks. In this robustness analysis, we are particularly interested in the process of how network performance degrades, so that remedies can be introduced to minimize the impact on network operations under such challenges. A highly robust (or resilient) network would show (almost) no degradation effects, but maintain its performance even under any type of perturbations [21].

A meta-metric such as robustness however cannot be evaluated separately, but only with respect to a set of selected metrics that the system designer or end user is concerned with, such as application-level performability, availability, path diversity etc., and for which robustness then assesses the corresponding sensitivity. As a result of this meta-analysis, the approach taken in this paper is not limited to the investigation of particular metrics, but may rather be applied towards any type of metric that is quantifiable.

To abstractly illustrate the general procedure, consider the case of a metric $m$ which measures some property of a network system, ranging from metrics characterizing links, nodes and the topology up to metrics assessing the performance of application-level services provided by network nodes. For this discussion and in a later application in our framework, metrics may capture anything of interest, however we make the fundamental assumption that any property may be captured at a particular point of time $t$ as a concrete value, so $m_{t} \rightarrow \mathbb{R}$. Depending on the metric, additional inputs and contextual information will be required, which can be specified as parameter values to $m$ : metrics such as connectivity or betweenness [22] require a representation of the network topology which is commonly specified though the graph $G=(V, E)$, so $m_{t}(G) \rightarrow \mathbb{R}$, a metric such as availability additionally uses knowledge about the unavailability of each network element $E \in V \cup E$ such as MTBF and MTTR, so $m_{t}\left(G,\left\{E_{k},\{M T B F, M T T R\}\right\}\right) \rightarrow \mathbb{R}$.

Since we are concerned about the resilience of a network and its robustness to stress, we investigate the metrics' change in value over time, when the network system is exposed to challenges, such as the (partial) failure of a link, node or specific function within each node. Using infinitely small time intervals, each challenge can be dissected into atomic 


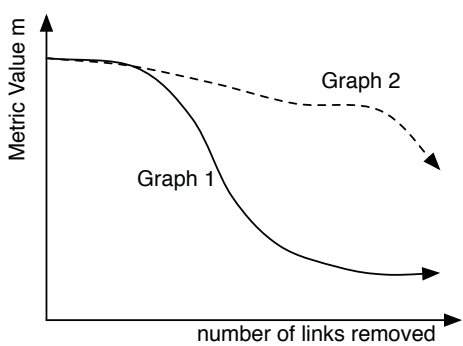

(a)

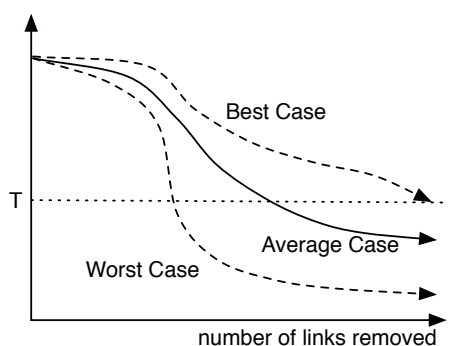

(b)

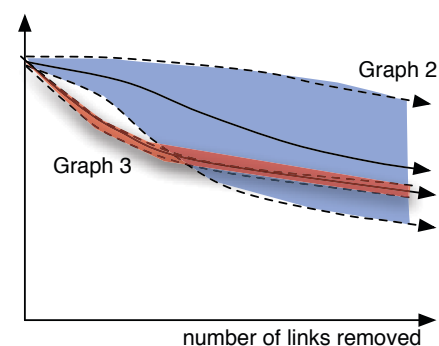

(c)

Fig. 1. To gain a comprehensive insight into the robustness of a network, best/worst/average network response behaviors need to be collected, resulting in an envelope that indicates the amount of "uncertainty".

perturbations that occur sequentially, so that between time $t$ and $t+1$ only one component has failed and the difference between $m_{t}(G)$ and $m_{t+1}(G)$ exactly captures the impact of the component (link, node or node function) that failed at time $t$. This allows us to (a) attach a concrete performance value as expressed by $m$ to every possible network state and (b) investigate how large and wide-scale failures spread throughout the system.

Using this procedure, an analysis as shown in figure 1(a) can be derived that captures the development of a particular metric $m$ as subsequently more and more components (in the figure for example links) fail. It is clear that this process of degradation will heavily depend upon the system parameters, as for example the configuration of nodes, the applications and services or the topological structure of the network considered in the evaluation.

To use a concrete example for the level of the (reasonably) well-understood topological metrics, it is known that a scalefree topology such as the Internet would be able to withstand random failures much better than for example homogeneous topologies such as an Erdös-Renyi (ER) graph [23] and still remain a connected network, as the random outages would over-proportionally strike at the highly connected hubs which simultaneously have a higher level of redundancy. The degradation of the corresponding metric connectivity would therefore be significantly less in absolute terms and relatively smoother for a network organized according to a scale-free paradigm than a homogeneous layout, as indicated by the development of $m$ for graph 2 and graph 1 in figure 1(a).

While one network topology would provide better robustness in terms of connectivity than another for random equipment failures, the prospects however immediately change when we look at other challenges that affect some parts of the network more than others or are even driven by malicious attackers targeting weak spots. It becomes evident that a comprehensive analysis of robustness must therefore go beyond an analysis of random failure events, but explore the overall network state space to find those chains of perturbations than will lead to the worst (and best) case impact to the system in terms of a particular metric. The envelope spread out between these two extremes, the best and worst case chain of perturbations (see figure 1(b)), will indicate what amount of risk and uncertainty there exists for a particular setup in terms of a particular metric, i.e., the minimum and maximum impact when encountering a challenge and the amount of possible variation in impact depending what kind of event took place. Such envelopes may be additionally refined and better risk assessments created if it can be determined a priori what classes of challenges (such as storms, floods, earthquakes, DDOS, worms etc.) a system will face; if a particular challenge class is not encountered during use, the resulting envelope may become smaller and the density of the network states is differently shaped.

We believe that in order to obtain a deep insight into network robustness, a quantification of the network state space according to the procedure outlined above will prove to be a substantial help due to its capability to determine hard and provable performance guarantees and help assessing the risk and uncertainty associated with a particular configuration when exposed to certain challenges. This point is further shown in figure 1(c), which illustrates that through the discussed best-average-worst envelopes a very meaningful comparison between network topology, configuration and design alternatives can be achieved. Depending on the use case and operating conditions of the system, an in absolute terms worse performing alternative such as graph 3 in figure 1(c) might actually be superior (a) if the network environment is for example expected to be subjected to a significant amount of malicious attacks or (b) if the operator intends to bound the network's variability as much as possible, for example when the system in question is part of a larger environment operating within tight boundaries. The computational framework described in this paper simplifies and automates this process by letting the network designer and operator specify which measurements, metrics and statistics, ranging from pure topological metrics to performance and service metrics at the application layer, are of particular interest and generate based on a specification of challenges at a multi-level, multidimensional analysis, pointing out which weak spots violate specified performance boundaries, providing an interface to optimize network design and operation. 


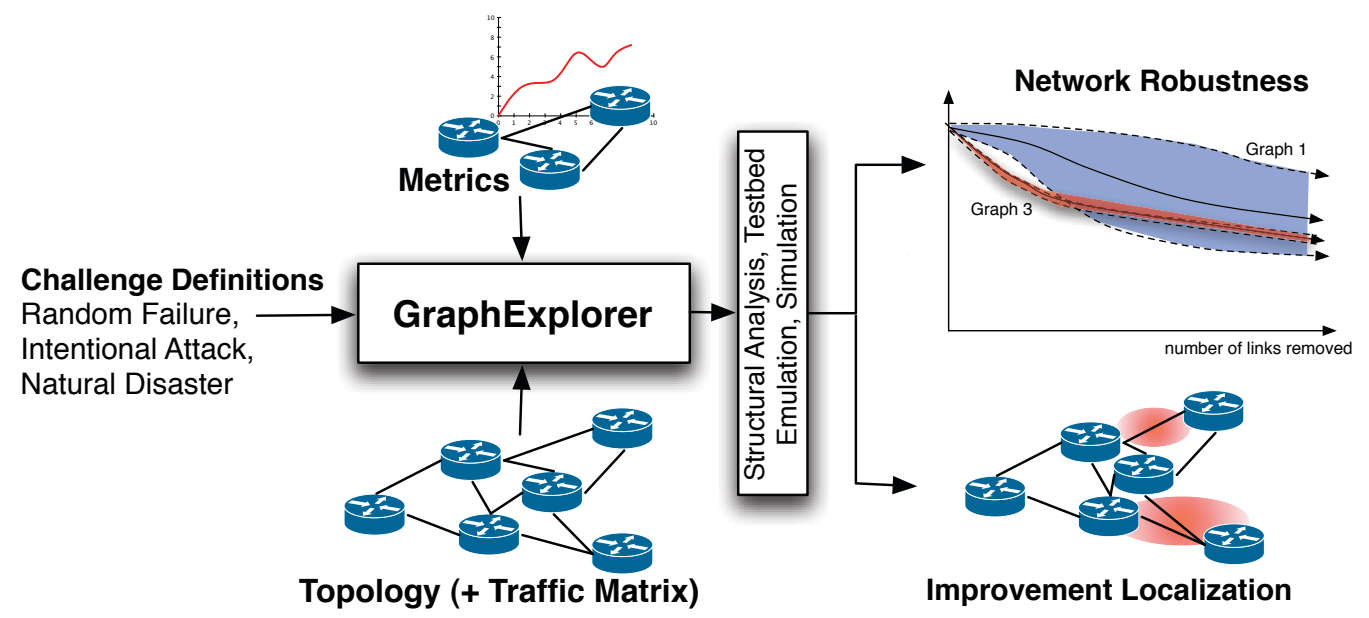

Fig. 2. The software described in this paper explores the robustness of a network system as measured by a set of metrics for a given set of challenges using structural (topological) analysis, testbed emulation or simulation. This will result in an overall performance evaluation as well as an indication of "weak spots".

\section{A COMPUTATIONAL FrAMEWORK FOR NETWORK RESILIENCE}

After the previous section described our conceptual approach to assessing network resilience, risks and uncertainty, this section will highlight a selection of key aspects of the actual software implementation, which provide the framework with sufficient versatility and reduce the computational complexity to meet the conceptual goals outlined above.

To achieve a cost-efficient and sustainable development, the entire software package was designed to be highly modular and reuse internal analysis components and externally available standard tools and packages as much as possible. Figure 2 shows an architectural overview of the system presented in this paper, which uses a combination of a topology description, a list and definitions of evaluation metrics, a set of challenges and their specifications, and if available a network traffic matrix and functional specification of the node behavior as input.

According to the metrics selected the system will use either analytical or structural exploration, software simulation, hardware emulation or a combination of these to evaluate each metric for all relevant network states; metrics such as availability or all topological metrics can be evaluated internally using the corresponding modules which define these metrics, while others such as delay, queuing statistics or application performance measures such as MOS values are being retrieved through external interfaces linking to an NS2 simulator or for example in our case a network testbed, emulating each scenario in hardware. Result of the state exploration is a series of envelope plots as discussed in section III, one for each evaluation metric, which may be overlayed, correlated, and specific degradation events meshed with a root cause analysis from the graph exploration. An additional output is an improvement localization map pinpointing those parts of the system leading to extraordinarily high performance degradation effects.

\section{A. Evaluating Scenarios}

As discussed in the previous section, the challenge definition specifies expectations and constraints on the possible localization, impact and frequency of failures. Based on these definitions and the underlying topology, the graph explorer then determines the entire state space of the system and determines an evaluation plan that will explore it using the least amount of computational resources.

As current network attack description languages such as IDMEF [24] or STATL [25] are targeted towards packet or session level attack scenarios used by anomaly or intrusion detection systems (IDS) and are therefore unsuited to model macroscopic challenges to a network such as random failures, natural disasters or intentional attacks, we developed two types of interfaces to specify challenges within the GraphExplorer framework: first, a Java API through which challenges and their impact on the network infrastructure can be directly implemented as part of the system, and second, an XMLbased description language to specify key characteristics of the challenge, such as where it may appear and how it will affect the network system.

While a complete discussion of the interfaces is without the scope of this paper, the following XML excerpt demonstrates the concept by specifying the example of a "storm challenge", which affects only wireless links in a network. The storm has a radius of $50 \mathrm{~km}$, may occur in certain parts of the topology and will (unrealistically) affect a link only if a wireless endpoint will be within the storm's range. In that case, the throughput will be decreased according to the $\sin ()$ of the terminal's distance to the center of the storm:

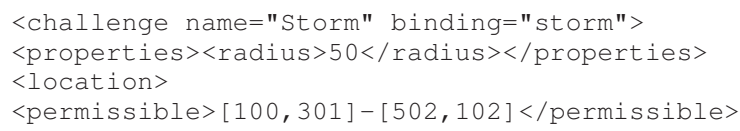




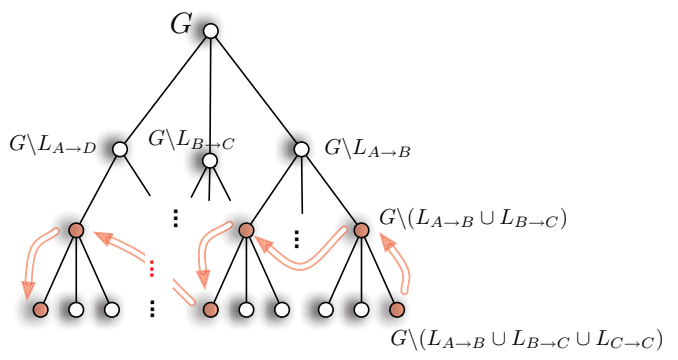

Fig. 3. The challenge specifications provided will guide the exploration of the possible network state space, so that only those concrete topology failure scenarios will be evaluated that may actually occur (indicated in red).

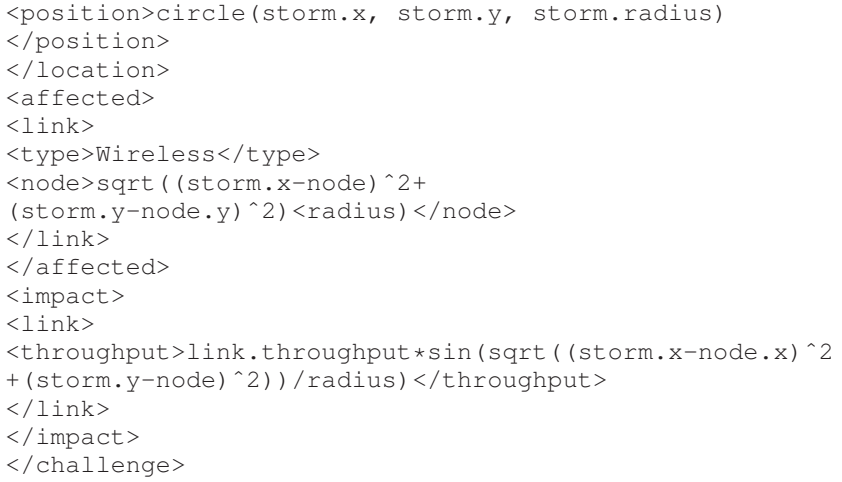

Using such an XML specification or a Java API implementation of a challenge, the system will then explore all network states that could potentially occur, i.e., for the challenge of a storm impacting wireless links specified above, it will enumerate all possible failure configurations where wireless links are affected in the network. Unless the network's resilience against any conceivable attack pattern needs to be evaluated (which then requires a full exploration of the graph state space), the state space may be heavily pruned as visualized in figure 3, as the impact of challenges is typically locally constrained (as in the example of the storm) and not an unbounded number of challenges may occur simultaneously. This makes the state exploration to derive hard best/worst case guarantees computationally tractable; the evaluation of a challenged wireless GEANT2 topology as presented in section $\mathrm{V}$ uses about 3 minutes on a workstation.

\section{B. Reducing Computational Complexity}

To further improve the response time of this way of resilience assessment, this section describes a series of simple yet effective architectural improvements to the graph exploration process as well as algorithmic improvements to the metrics themselves and their computation that significantly reduce the computational demand required and will consequently make the quantification of uncertainty and exploration of best and worst cases possible even for moderately-sized network topologies.
The fundamental idea behind the graph exploration and also the fundament for all subsequent optimizations is an ordered run through the network state space, ordered in such a way that it minimizes the metric evaluation, reconfiguration and management overhead demand on a per step basis. Using this ordered list of network states as supplied by the graph explorer and the challenge definition, a depth first search (DFS) of this global ordering combined with the observation that network component failures are commutative - a failure of link $1 \rightarrow 2$ and subsequently $3 \rightarrow 4$ will have the same impact on network performance as $3 \rightarrow 4$ and $1 \rightarrow 2$ - will provide the most effective evaluation method for three reasons: first, only based on the queuing position in the DFS tree and without additional context information, it can be determined which next steps need to be explored and which parts of the tree may be pruned as the results can be inferred from previous evaluation runs. Second, a DFS based approach (in contrast to other state exploration strategies) will ensure that between each state only a single atomic perturbation took place, a requirement outlined in section III, which as shown later will greatly simplify the calculation of metrics, and third, from an algorithmic and software implementation point of view offers the most memory effective data organization requiring the least amount of context switches. While sounding rather trivial, the combination of the three together with a streamlined metric calculation cuts down the computational complexity by several orders of magnitude, making the in principle combinatorial problem solvable even for medium-sized network topologies. The risk analysis for a concrete challenge such as the storm for such a network requires less than 5 minutes on a normal workstation, while the results for a complete analysis including hard performance guarantees can be obtained in less than an hour. For larger instances the DFS approach allows for efficient parallelization.

In addition to being selective on which and how component failures are investigated, resources can also be saved by streamlining the operation of the metrics used throughout the analysis. In the following, we will discuss such an optimization for the metrics of average path length and node/link betweenness [22], which are widely used for graph analysis.

Both average path length, which measures the shortest distance between all node pairs in a graph, and betweenness, which captures how many shortest paths within a network are traversing a particular link or node en route to the destination, are basing their results on a calculation of the shortest paths within a topology from which they do further result aggregation. While the final aggregation step, e.g. averaging the length of the paths, cannot be improved by much, the actual calculation of the paths contains a number of improvement opportunities due to our very specific use case.

The best procedure for the underlying so-called all pairs shortest path (APSP) problem is the Floyd-Warshall algorithm [26], which by comparing and relaxing all pairs of nodes in a graph computes the result in a run time of $O\left(N^{3}\right)$. While a full exploration of the state space would be infeasible when it would be necessary to recompute such metric after each 


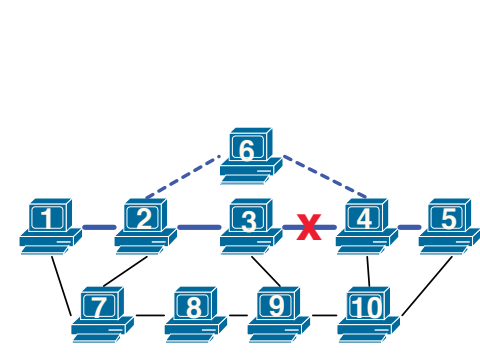

(a)

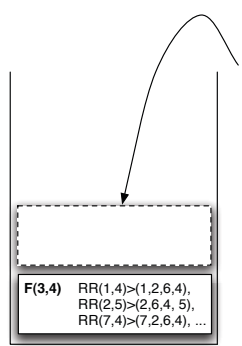

(b)
Fig. 4. Through specific exploration strategies, the search space and overall computation complexity may be drastically reduced.

perturbation to the graph, it becomes practical if we consider two features of the metric calculation and specific exploration strategy discussed in the previous sections that bound overall complexity:

1. If between two evaluations of the metric only one network component has failed (which is realized by our atomic exploration), only that part of the graph and its corresponding metric values need to be updated where the component has actually failed or where remote components are immediately affected by it. The assessment around this affected area and all related intermediate results remain unchanged and may be used for a quick recovery and update of the metric.

2. The relaxation step during the calculation of the 1dimensional shortest path problem assumes per induction that all subpaths of a shortest path are also optimal. This enables us that if a component along an optimal path has failed we can limit our search to only the upstream hops along this route (along which due to \#1 the results do not need to be recomputed).

Figure 4(a) visualizes this concept in a small example. The initial run of the Floyd-Warshall algorithm in $O\left(N^{3}\right)$ has determined all shortest paths between the nodes in this topology and implicitly identified where the shortest paths between node pairs run along, i.e., the link betweenness of the topology. Consider now our first incremental update, a link failure between nodes 3 and 4. From the previous (initial) computation, we can deduce that only those paths which contribute to the link betweenness of link $3 \rightarrow 4$, namely $1 \rightarrow 4$, $1 \rightarrow 5,2 \rightarrow\{4,5\}$, will be affected by this update and therefore need to be recomputed (assumption \#1).

W.l.o.g. we will show the recovery and updating procedure for the affected path $1 \rightarrow 5$, where we will refer to node 1 as source and node 5 as destination: Using the relaxation paradigm of shortest path calculations (\#2), we know that a path is only affected if it was indeed (one of) the shortest path between two nodes, otherwise no recomputation of the metric becomes necessary. From induction we also know that the next-best shortest path between the node pair will consequently branch off anywhere between the current failure point and the source and that its distance is at least as long as the component we are replacing. Traversing the links upstream we identify an alternative route at node 2 (through node 6) which replaces the subpath with the failed component in exactly the same number of links. In this situation the search may stop, as clearly no better path in the topology exists. In case a replacement relaxation of equal length cannot be found en route from the failure location to the source (i.e., in the example of Fig. 4(a) node 6 would not exist), the search progresses until it reaches the source and chooses the minimum of all relaxations encountered along the previous shortest path $([2 \rightarrow 7 \rightarrow \ldots \rightarrow 5]+[1 \rightarrow 2]=5+1=6,[1 \rightarrow 7 \rightarrow \ldots \rightarrow 5]=5)$.

Through such incremental calculation processes, the computational complexity can be drastically reduced. In the case of the average path length and betweenness metrics, the overhead may be reduced from $O\left(N^{3}\right)$ to a minimum of $O(k \cdot N)$ for graphs including some levels of path diversity and redundancy.

In addition to reducing the update complexity of a metric after a component failure, the described incremental update procedure can furthermore feed back and improve the general graph exploration discussed in the previous section. As it is known to the metric after each perturbation how a particular failure spread across the network and which parts were affected, this combined knowledge about failures, affected and rerouted paths can be saved on a stack as indicated in figure 4(b) and exploited during the future exploration: (1) if a component is repaired (in a remediation action or on the DFS backtrack) the original (then again) best shortest path is simply restored from the stack thereby eliminating any need for reevaluation, and (2) values for other perturbations that are in fact commutative graph operations to previously investigated situations can be looked up from this history. We have found that these two steps may additionally reduce the overall running time by half in practice.

\section{Use Case Study}

After the general concept of our resilience exploration, the quantification of risk and challenges have been discussed in the previous sections, this section presents a selection of results obtained from a resilience case study of the European research network GEANT2, demonstrating the benefit of such a unified approach. In this case study, we investigated the general vulnerability of this network deployment, specifically to random failures, an intentional simultaneous attack and the impact of a natural phenomenon such as a storm shorting out links if the GEANT network would be using long-distance wireless instead of fiber-optic links.

In the part of the case study presented in this paper, we investigated how an application level performance measure such as jitter would be affected if the network becomes challenged and link failures occur, which then forces extensive rerouting of network traffic and potential overloads at certain core routers. To better understand how the application level metric jitter (layer 7 in the ISO model) would be impacted, we followed the proposed multi-level, multi-dimensional approach and let the GraphExplorer simultaneously monitor a number of metrics at various levels of the network stack: the queue length of routers in the networks (layer 4), the current length of 


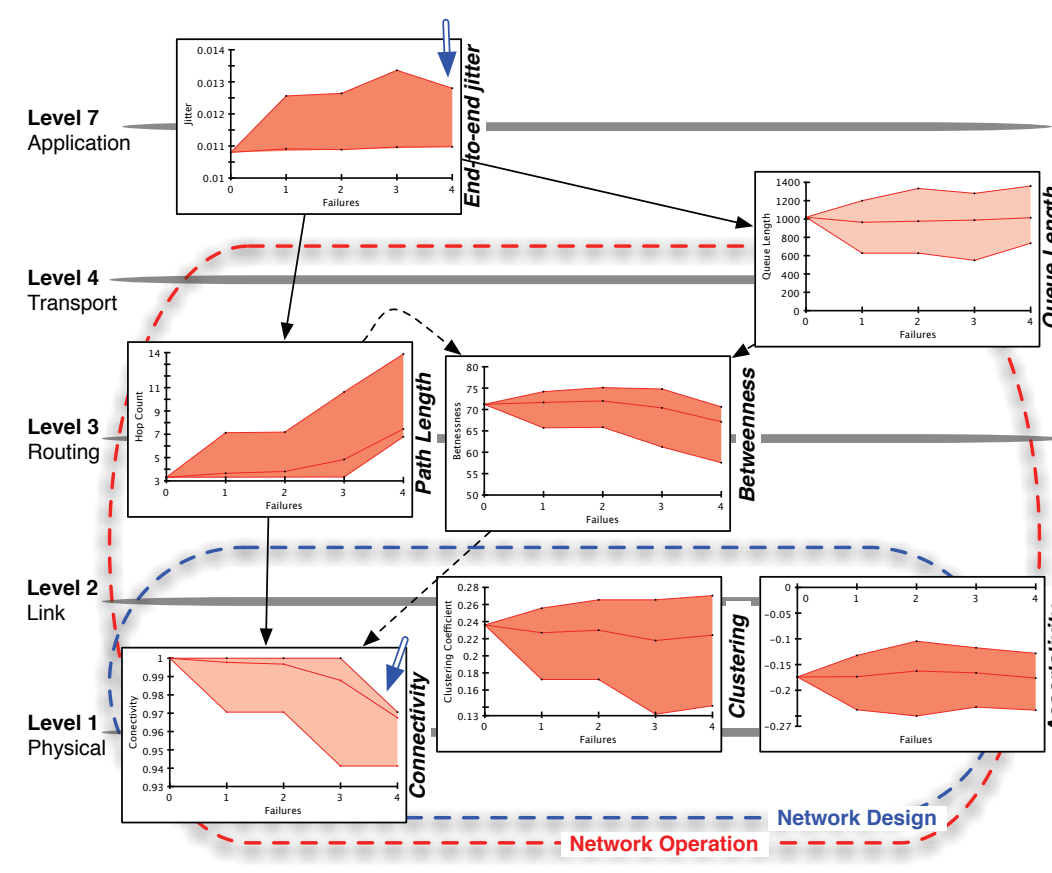

(a) A comparison of various network metrics both horizontally at a layer of the networking stack (b) The risk map indicates the most vulnerable parts and and vertically across layers may give a better insight into the root causes of performance degradation the largest impact to a hypothetical wireless GEANTto select the best possible remediation.

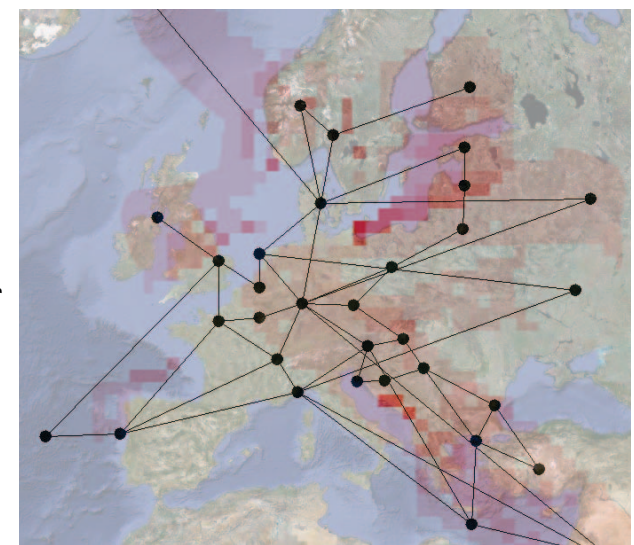
network if perturbed by a storm.

Fig. 5 .

network paths and the concentration of shortest paths on links, the betweenness, at layer 3 , the metrics clustering coefficient and assortativity monitoring the coherence and layout patterns of the network topology (layer 1/2) and the general availability of a links as captured through the metric connectivity (layer 1). As these metrics span the range from pure topological to pure application-level service metrics, the GraphExplorer uses a mixture of structural analysis and NS2 simulation to evaluate the specified measures.

Figure 5(a) shows a mapping of the performance envelopes of the seven metrics used in this case study on their particular position in the ISO networking stack, up to a failure depth of 4 , i.e., when 4 arbitrary links in the network may have failed. As can be seen in the embedded figures, the corresponding performance of each metric will degrade as the network becomes more and more challenged; the application-level jitter increases as does the length of the (now rerouted) network paths, while the physical connectivity and the availability of end-to-end paths on network links will decrease. It is worth noting that each link failure will not degrade the network to the same extent, in fact there is no significant difference whether one or two links have failed in the topology.

Conceptually, there clearly exist a number of dependencies between metrics, for example, it is reasonable to assume that the end-to-end jitter will be influenced by the length of the queues at the intermediate routers as well as by the average length of paths (number of hops) packets need to traverse towards the destination. Surprisingly however, the results obtained by the GraphExplorer from NS2 simulations do not indicate such clear dependencies and also indicate that while additional link failures will result in a monotonic increase in path lengths in the network, this will not necessarily increase queue lengths and ultimately end-to-end jitter. In fact, after the fourth link has failed, the jitter as experienced by the applications using the network will actually decrease (see arrow in jitter envelope inset).

To help investigate the root cause for this observation, the GraphExplorer points out a variety of relationships and correlations that were found during the evaluation of the failure cases of this particular scenario as indicated by the dashed arrows, (1) a relationship between the queue length and the betweenness of links and assortativity of nodes, (2) a dependence of the path length on link betweenness and (3) a relationship between the link betweenness and the physical connectivity of the topology, with the latter two showing at $r^{2}=0.9$ a near complete dependence. By using the best, average, and worst case envelopes and these correlation figures, it is now possible to explain the seemingly strange decreasing application jitter after the fourth link failure. As the connectivity envelope indicates, even in the best possible failure scenario will a fourth link cut disconnect a subset of the network (see arrow in connectivity envelope inset). The disconnect will occur at the previous bottleneck node, which after three link failures provided the only connection between the two components, thus carrying all traffic between the subnetworks and therefore experiences large queues and 
introducing high jitter. After the fourth failure, as some nodes of the network are now disconnected from the core, the path length will subsequently increase as the routes to the unreachable destinations are set to $M A X_{-} H O P S$, however the decrease in the betweenness' envelope clearly indicates that this increase is due to the loss of certain routes. As these paths are not traversed anymore due to unreachability of the destination, the former bottleneck router now becomes a normal, less-loaded edge node, leading to a decrease in jitter as experienced by the nodes in the network.

After understanding how physical link failures at layer 1 ultimately lead to a decrease in end-to-end jitter at layer 7, these insights can be used to design adaptations and remedies to make the network topology more robust. If we conduct such kind of investigation during the network design phase before an actual deployment has happened, we could for example change the underlying physical topology of the network (layer $1 / 2$ ) to create a more resilient system. After deployment and during network operation, different remediation actions might be taken, ranging from a change of the queuing policy to preempt real-time traffic (layer 4 ), create MPLS routes to balance traffic flows and avoid single bottlenecks (layer 2.5/3) to temporarily adding leased lines (layer 1/2).

It can further be determined from this type of analysis where such remediation actions would be most beneficial to network operation. Figure 5(b) shows a risk map which indicates the most vulnerable parts and possible improvement areas, here for the case of a hypothetical wireless GEANT network challenged by a large-scale storm, which would degrade if not eliminate the network links within its range, thus causing reroutes, traffic overloads and finally throughput degradations. The intensity of the red color displays the severity of the perturbation. If challenges are not geographically but conceptually defined, such as simultaneous terrorist attacks against e.g. routers or fibers, the corresponding risk map would colorcode those combinations of network resources whose failure would be most severe to the network operation as specified by the metrics.

\section{CONCLUSIONS}

This paper discussed a novel computational approach for multi-level, multi-dimensional robustness assessment of network systems, aiming to bring together and jointly use various proposals, techniques and approaches from the scientific community. By viewing many metrics at various levels simultaneously, better inferences about the root causes and spread of a failure event can be made and better remediations may be designed. Through this approach, it is possible to derive provable best and worst case performance bounds of a network's resilience and quantify the uncertainty and risk associated with particular challenge scenarios. The approach has been successfully tested in several case studies and the results which can be generated in comparable time as for example through mathematical programming approaches have been validated where applicable through other research groups.
Acknowledgements The work presented in this paper is supported by the European Commission, under Grant No. FP7224619 (the ResumeNet project).

\section{REFERENCES}

[1] V. J. Bono, "7007 explanation and apology, http://www.merit.edu/ mail.archives/nanog/1997-04/msg00444.html,” 1997.

[2] RIPE, "Youtube hijacking: A ripe ncc ris case study, http://www.ripe.net/news/study-youtube-hijacking.html," 2008.

[3] T. Duebendorfer, A. Wagner, and B. Plattner, "An economic damage model for large-scale internet attacks," in IEEE Workshop on Enabling Technologies, 2004.

[4] R. Cohen, K. Erez, D. ben Avraham, and S. Havlin, "Breakdown of the internet under intentional attack," Phys. Rev. Let., vol. 86, 2001.

[5] O. Nordström and C. Dovrolis, "Beware of bgp attacks," SIGCOMM Comput. Commun. Rev., vol. 34, no. 2, pp. 1-8, 2004.

[6] Z. M. Mao, R. Govindan, G. Varghese, and R. H. Katz, "Route flap damping exacerbates internet routing convergence," in Proceedings of SIGCOMM, 2002.

[7] L. da F. Costa, F. A. Rodrigues, G. Travieso, and P. R. V. Boas, "Characterization of complex networks: A survey of measurements," Advances in Physics, vol. 56, no. 1, 2007.

[8] M. Menth, M. Duelli, R. Martin, and J. Milbrandt, "Resilience analysis on packet-switched communication networks," IEEE/ToN, vol. 99, 2009.

[9] P. Cholda, A. Mykkeltveit, B. E. Helvik, O. J. Wittner, and A. Jajszczyk, "A survey of resilience differentiation frameworks in communication networks," IEEE Comm. Surveys, vol. 9, no. 4, pp. 32-55, 2007.

[10] H. L. Frisch and J. M. Hammersley, "Percolation processes and related topics," SIAM Journal on Applied Mathematics, vol. 11, no. 4, pp. 894918, 1963.

[11] M. F. Sykes and J. W. Essam, "Exact critical percolation probabilities for site and bond problems in two dimensions," Journal of Mathematical Physics, vol. 5, no. 8, pp. 1117-1127, 1964.

[12] M. Chari and C. J. Colbourn, "Reliability polynomials: A survey," $J$. Combin. Inform. System Sci, vol. 22, pp. 177-193, 1998.

[13] S.-C. Chang and R. Shrock, "Reliability polynomials and their asymptotic limits for families of graphs," J. Stat. Phys., vol. 112, p. 1019, 2003.

[14] L. Page and J. Perry, "Reliability polynomials and link importance in networks," Reliability, IEEE Transactions on, vol. 43, pp. 51-58, Mar 1994.

[15] P. Holme, B. J. Kim, C. N. Yoon, and S. K. Han, "Attack vulnerability of complex networks," Physical Review E, vol. 65, p. 056109, 2002.

[16] D. S. Callaway, M. E. J. Newman, S. H. Strogatz, and D. J. Watts, "Network robustness and fragility: Percolation on random graphs," Phys. Rev. Let., vol. 85, p. 5468, 2000.

[17] M. Faloutsos, P. Faloutsos, and C. Faloutsos, "On power-law relationships of the internet topology," in In SIGCOMM, pp. 251-262, 1999.

[18] M. Cushing, J. Krolewski, T. Stadterman, and B. Hum, "U.S. army reliability standardization improvement policy and its impact," IEEE Trans. on Components, Packaging, and Manufacturing Technology, vol. 19, pp. 277-278, 1996.

[19] I. Gashi, P. Popov, and L. Strigini, "Fault tolerance via diversity for off-the-shelf products: A study with sql database servers," IEEE Trans. Dependable Secure Comput., vol. 4, pp. 280-294, Oct.-Dec. 2007.

[20] L. Huang, K. Yang, and L. Yang, "Enhancing robustness and immunization in geographical networks," Physical Review E (Statistical, Nonlinear, and Soft Matter Physics), vol. 75, no. 3, p. 036101, 2007.

[21] J. Stelling, U. Sauer, Z. Szallasi, F. J. D. III, and J. Doyle, "Robustness of cellular functions," Cell, vol. 118, no. 6, pp. 675 - 685, 2004.

[22] L. Freeman, "A set of measures of centrality based upon betweenness," Sociometry, vol. 40, pp. 35-41, 1977.

[23] P. Crucittia, V. Latora, M. Marchiori, and A. Rapisarda, "Error and attack tolerance of complex networks," Physica A, vol. 340, pp. 388-394, 2004.

[24] D. Curry, "Intrusion detection message exchange format: Extensible markup language (xml) document type definition.'

[25] S. T. Eckmann, G. Vigna, and R. A. Kemmerer, "Statl: An attack language for state-based intrusion detection," Journal of Computer Security, vol. 10, pp. 71-103, 2002.

[26] R. W. Floyd, "Algorithm 97: Shortest path," Comm. of the ACM, vol. 5, no. 6 , p. 345,1962 . 